\title{
High Repetition-Rate Neutron Generation by Several-mJ, 35 fs pulses interacting with Free-Flowing $\mathrm{D}_{2} \mathrm{O}$
}

\author{
J. Hah, ${ }^{1,2}$ G. M. Petrov, ${ }^{3}$ J. A. Nees, ${ }^{1,4}$ Z-H. He, ${ }^{1,2}$ M. D. Hammig, ${ }^{2}$ K. Krushelnick, ${ }^{1,2,4}$ and A. G. R. \\ Thomas ${ }^{1,2,5}$, a) \\ ${ }^{1)}$ Center for Ultrafast Optical Science, University of Michigan, Ann Arbor, MI 48109 USA \\ ${ }^{2)}$ Department of Nuclear Engineering and Radiological Sciences, University of Michigan, Ann Arbor, \\ MI 48109 USA \\ ${ }^{3)}$ Naval Research Laboratory, Plasma Physics Division, 4555 Overlook Ave. SW, Washington, DC 20375, \\ USA \\ ${ }^{4)}$ Department of Electrical Engineering and Computer Science, University of Michigan, Ann Arbor, \\ MI 48109 USA \\ ${ }^{5)}$ Department of Physics, University of Michigan, Ann Arbor, MI 48109 USA
}

(Dated: 10 May 2016)

Using several-mJ energy pulses from a high-repetition rate $(1 / 2 \mathrm{kHz})$, ultrashort (35 fs) pulsed laser interacting with $\mathrm{a} \sim 10 \mu \mathrm{m}$ diameter stream of free-flowing heavy water $\left(\mathrm{D}_{2} \mathrm{O}\right)$, we demonstrate a $2.45 \mathrm{MeV}$ neutron flux of $10^{5} / \mathrm{s}$. Operating at high intensity (of order $10^{19} \mathrm{Wcm}^{-2}$ ), laser pulse energy is efficiently absorbed in the pre-plasma, generating energetic deuterons. These collide with deuterium nuclei in both the bulk target and the large volume of low density $\mathrm{D}_{2} \mathrm{O}$ vapor surrounding the target to generate neutrons through $\mathrm{d}(d, n)^{3} \mathrm{He}$ reactions. The neutron flux, as measured by a calibrated neutron bubble detector, increased as the laser pulse energy was increased from $6 \mathrm{~mJ}$ to $12 \mathrm{~mJ}$. A quantitative comparison between the measured flux and results derived from 2D particle-in-cell simulations show comparable neutron fluxes for similar laser characteristics to the experiment. The simulations reveal that there are two groups of deuterons; forward moving deuterons generate $\mathrm{D}-\mathrm{D}$ fusion reactions in the $\mathrm{D}_{2} \mathrm{O}$ stream and act as a point source of neutrons, while backward moving deuterons propagate through the low-density $\mathrm{D}_{2} \mathrm{O}$ vapor filled chamber and yield a volumetric source of neutrons.

Energetic neutrons have numerous applications in many fields, including medicine ${ }^{1}$, homeland security ${ }^{2}$, and material science ${ }^{3}$. Conventional fast neutron sources include deuterium-deuterium (D-D) and deuteriumtritium (D-T) fusion generators, as well as light-ion, photoneutron and spallation sources. Laser plasma interactions in the relativistic regime can also generate charged particles and subsequently accelerate them to energies high enough to trigger nuclear fusion reactions, resulting in neutron production ${ }^{4-16}$. Recent advances in ultra-high power laser technology now enable tabletop scale systems, which may be further reduced in size for use as drivers for portable neutron generators in the future. One of the methods for neutron production is through the acceleration of high-energy ions (keV-MeV) impinging upon an appropriate converter target, such as deuterated plastic. Typically, thin solid targets are used in these experiments to accelerate deuterons.

Using solid targets in the form of a thin $(1 \mu \mathrm{m})$ foil has some drawbacks for high repetition-rate $(>\mathrm{kHz})$ operation; for example, one has to replace the target after each shot. To resolve target life-time issues, fast target replacement schemes have been introduced by some groups $^{8,15,17-19}$. In particular, using $\sim 100 \mathrm{~mJ}$ of pulse energy at $10 \mathrm{~Hz}$ repetition-rate, Ditmire et. al. ${ }^{8}$ used deuterium clusters, which were rapidly heated by the laser pulse (on a femtosecond time scale) and launched few

\footnotetext{
a) Now at Physics Department, Lancaster University, UK
}

$\mathrm{keV}$ deuterons to drive $\mathrm{D}-\mathrm{D}$ fusion reactions.

In this Letter, we report the production of neutrons using a high repetition-rate $(1 / 2 \mathrm{kHz})$ femtosecond laser at high intensities ( $>10^{19} \mathrm{Wcm}^{-2}$ for vacuum focus) but low pulse energies (several-mJ) interacting with a heavy water stream. We demonstrate a conversion efficiency of laser energy into $2.45 \mathrm{MeV}$ neutrons of $\sim 10^{-8}$, which is comparable to previous experiments that utilized tabletop systems ${ }^{8,16,17,19-21}$.

Our experiment uses the Lambda-cubed $\left(\lambda^{3}\right)$ laser facility at the University of Michigan. The $\lambda^{3}$ laser is a Ti:sapphire system $(\lambda=800 \mathrm{~nm})$ producing laser pulses of duration $\tau=35 \pm 2$ fs full-width-at-half-maximum (FWHM) with an amplified-spontaneous-emission (ASE) intensity contrast ratio of $10^{8}$. However, for these experiments we deliberately introduce a pre-pulse split from the main pulse arriving $13 \mathrm{~ns}$ before the main pulse with an intensity contrast ratio of $10^{3}$. This pre-pulse serves to generate a pre-plasma and increase the absorption of the main pulse energy.

The $\lambda^{3}$ laser operates at a $1 / 2 \mathrm{kHz}$ repetition rate and delivers up to $18 \mathrm{~mJ}$ energy per pulse focused upon heavy water stream targets. The laser was focused to a $1.3 \mu \mathrm{m}$ FWHM focal spot using an $f / 1.4$ off-axis parabolic mirror, which produced a maximum peak intensity of $3 \times 10^{19}$ $\mathrm{W} / \mathrm{cm}^{2}$ in vacuum. A commercial syringe pump (Teledyne Isco) was used to maintain a constant flow rate of heavy water through a $10 \mu \mathrm{m}$ capillary, which produced a $15 \mu \mathrm{m}$ diameter continuous flow of either heavy $\left(\mathrm{D}_{2} \mathrm{O}\right)$ or light $\left(\mathrm{H}_{2} \mathrm{O}\right)$ water, with a flow rate of $100 \mu \mathrm{L} / \mathrm{min}$. The laser focus was set approximately $300 \mu \mathrm{m}$ below the 
tip of the capillary and at the first surface normal to the flowing water. The chamber pressure was maintained at 20 Torr during the experiment by a roughing pump. Heavy water vapor originating from the stream filled the chamber and therefore acted as a catcher for the accelerated deuterons for neutron generation. The experimental setup is shown in Fig. 1. Second harmonic light reflected from the stream and off the parabolic mirror passed out of the chamber and was used to guarantee a normal incidence focus on the stream's surface. A CdTe x-ray detector was then used to precisely optimize this alignment to produce x-ray counts on every single shot. Fine target alignment was performed using piezo actuators (Newport Picomotor) for xyz-control of the paraboloid and the water stream.

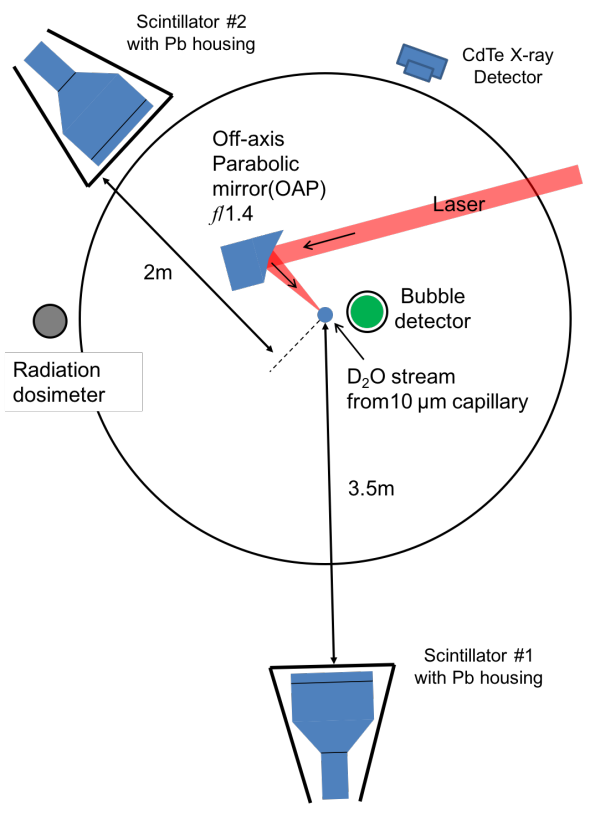

FIG. 1. Experimental setup. Note that scintillator distances are not to scale, and the chamber diameter is $40 \mathrm{~cm}$, and the chamber is filled with 20 Torr of heavy water vapor.

In order to confirm the production of neutrons from D-D fusion reactions, we employed two different detection schemes. We used several bubble detectors (Bubble Tech Industries, BD-PND) located $4 \mathrm{~cm}$ from the interaction region at various angles with respect to the laser propagation direction, with the data shown in this Letter being taken at $45^{\circ}$. These bubble detectors contain superheated droplets $(20 \mu \mathrm{m}$ to $50 \mu \mathrm{m}$ in diameter $)$, which vaporize into macroscopic bubbles $(0.2 \mathrm{~mm}$ to $0.5 \mathrm{~mm}$ in diameter) when they are irradiated by neutrons. In the energy range between $0.3 \mathrm{MeV}$ and $10 \mathrm{MeV}$, the BD-PND detector has a flat response ${ }^{22}$ and exhibits good linearity with respect to neutron dose $\mathrm{e}^{23}$. Most importantly, these bubble detectors are insensitive to both x-rays and electron interactions. The bubble detector was also covered with a $1 \mathrm{~mm}$ thick aluminum tube to protect it from direct laser irradiation.

Our bubble detector was independently calibrated for $2.45 \mathrm{MeV}$ neutrons using a commercial neutron generator at the Neutron Science Laboratory at the University of Michigan (MP-320, Thermo Scientific). The $10^{6}$ neutrons/sec neutron flux of the source yielded a calibration factor for the bubble detector of 6900 neutrons per bubble. Exposure of the bubble detector to the laserbased neutron source for 2 minute accumulation periods resulted in the formation of up to 40 bubbles, corresponding to a calibrated neutron flux on the order of $10^{5}$ neutrons/sec.

A second detection scheme consisted of two plastic scintillators (ELJIN, EJ-204) coupled to photo-multiplier tubes (HAMAMATSU, H2431-50 biased at $-2.5 \mathrm{kV}$ ). Using the plastic scintillators, neutron time-of-flight analysis was performed to determine the neutron energy spectrum. Two scintillators located at different distances and directions (2 m, $3.5 \mathrm{~m}$, as in Fig. 1), measured the neutron time-of-flight signal. Two such recorded signals are shown in Fig. 2. Results from heavy water (black and red curves) were recorded at the stated distances and the light water signal (blue curve) was recorded independently at $3.5 \mathrm{~m}$ as a control. The heavy water traces are the pulse shapes averaged over the 300 measurements that exceed the $2 \mathrm{mV}$ noise floor in the neutron interval, while the light water trace shows the pulse shape averaged over the 4000 measurements that exceed the $2 \mathrm{mV}$ noise floor in the whole window.

All traces have x-ray peaks starting near $0 \mathrm{~s}$, but only the two heavy water traces possess secondary peaks (black and red curves) corresponding to the relatively slow transit of the fusion neutrons to the detector following their creation. If $\mathrm{D}-\mathrm{D}$ fusion occurs at the $\mathrm{D}_{2} \mathrm{O}$ target and fusion neutrons propagate without scattering into the detector, then the resulting $2.45 \mathrm{MeV}$ neutron will interact with the scintillator $92 \mathrm{~ns}$ and $162 \mathrm{~ns}$, for the $2 \mathrm{~m}$ and $3.5 \mathrm{~m}$ separations, respectively. However, the peaks for the $2 \mathrm{~m}$ and $3.5 \mathrm{~m}$ cases are observed at $110 \mathrm{~ns}$ and $182 \mathrm{~ns}$, instead, showing that both signals are delayed by up to 20 ns from the expected arrival times. The effect of the delayed transit-time produces the downshifted and broadened neutron energy spectra shown in the inset of Fig. 2. The delay is due to both scattering and neutron generation occurring at a distance from the water stream as $\mathrm{D}^{+}$ions interact with $\mathrm{D}_{2} \mathrm{O}$ vapor within the vacuum chamber, as will be detailed in the numerical simulation results shown below.

The $\mathrm{D}_{2} \mathrm{O}$ stream will not have sharp boundaries predominantly because the main laser shot is preceded by a pre-pulse ${ }^{24-28}$, which ablates the material and generates a pre-plasma surrounding the water target. The creation of this pre-plasma is beneficial as it increases the coupling efficiency of the laser energy to the production of hot electrons, whose improvement enhances the energy of the deuterons. 


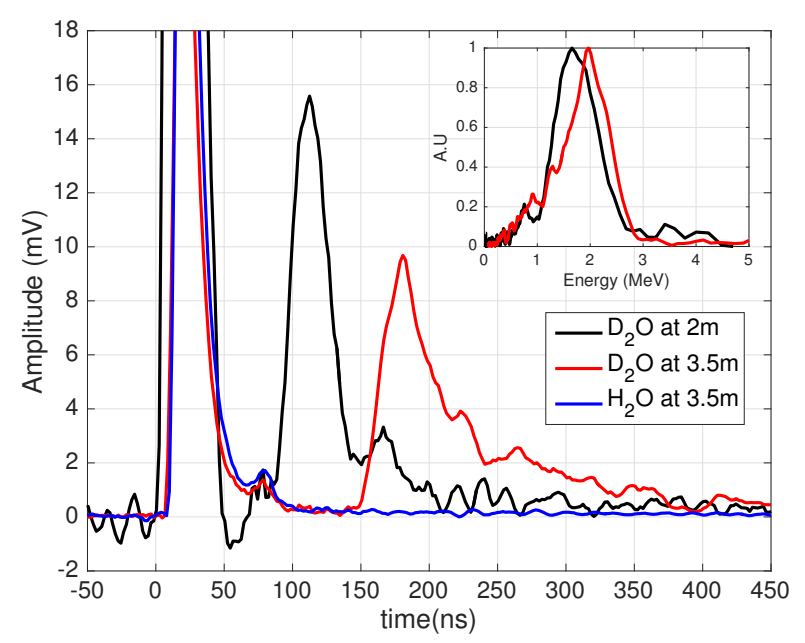

FIG. 2. Neutron time-of-flight analysis. The voltage signal derived from the PMT following each x-ray interaction is averaged over 300 traces. The average trace derived from the light water target, shown in blue, is compared to the average traces derived from the heavy water target, when the neutron and $\mathrm{x}$-ray sensitive scintillator is separated from the target by $2 \mathrm{~m}$ and $3.5 \mathrm{~m}$, respectively. The inset shows the neutron energy spectrum derived from the approximate time-of-flight measurements.

Numerical simulations were performed using a twodimensional electromagnetic particle-in-cell code ${ }^{29,30}$. The $\mathrm{D}_{2} \mathrm{O}$ target was approximated as a planar target at liquid density, having a pre-plasma with density exponentially decreasing away from the edge with a characteristic length of $1 \mu \mathrm{m}$ at $1 / e$. This pre-plasma scale length is estimated from measurements with a similar laser system interacting with water targets ${ }^{31}$. With an assumed focal spot size $\left(\mathrm{D}_{F W H M}\right)$ of $1 \mu \mathrm{m}$ and a pulse duration $\left(\tau_{F W H M}\right)$ of $32 \mathrm{fs}$, the laser-water interaction was simulated for peak laser intensity variations between $1 \times 10^{19} \mathrm{~W} / \mathrm{cm}^{2}$ and $3 \times 10^{19} \mathrm{~W} / \mathrm{cm}^{2}$.

The on-target laser energy was varied between 3.7 and $11 \mathrm{~mJ} /$ shot. Particles were initialized with charge +1 for ions and -1 for electrons and during the simulations the ion charge of oxygen was dynamically incremented using a standard Monte Carlo scheme for collisional and optical field ionizations. At the beginning of the simulations, the number of particles-per-cell was 36 for deuterons and 18 for oxygen ions. The momentum distribution of deuterons is plotted in Fig. 3 .

The phase-space plot reveals the dynamics of the interaction. First, the laser interacts primarily with the pre-plasma and all of the energetic ions originate from the pre-plasma region. The second intriguing observation is that there are two distinct groups of deuterons: one group accelerated forward, and another group in the backward direction (towards the incoming laser direction). Their number is comparable, but the backward moving deuterons are more energetic. In the

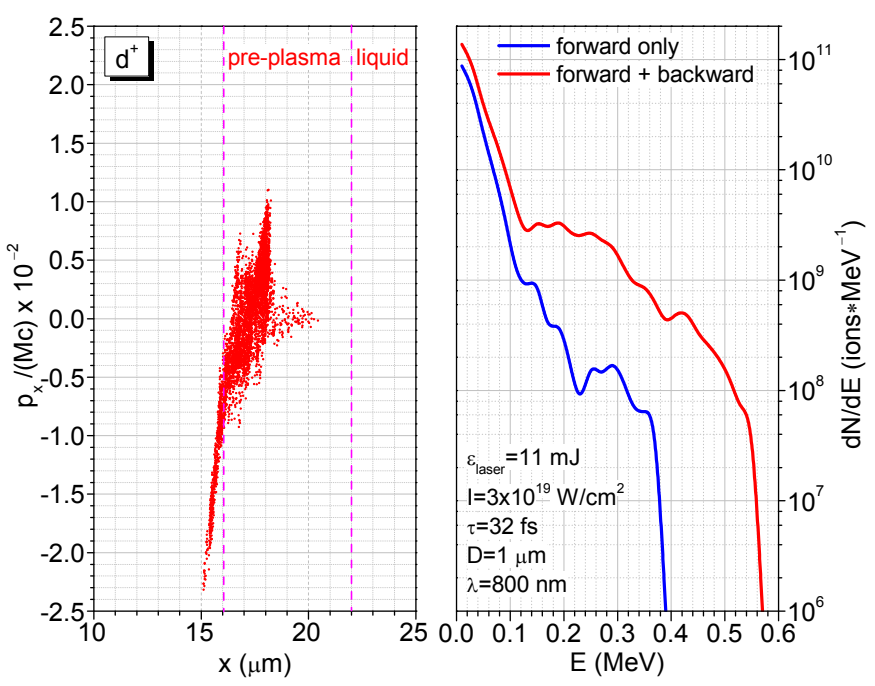

FIG. 3. (Left) Deuteron momentum $x-p_{x}$ phase space. (Right) Deuteron energy spectrum.

forward direction the maximum normalized impulse is $\mathrm{p}_{x} /(\mathrm{Mc}) \sim 0.01$. The corresponding momentum in the backward direction is double.

Neutron production is very sensitive to the direction of the deuterons. Forward-directed deuterons see increasingly dense plasma, all the way up to liquid density in the stream. Since their range is only a few $\mu \mathrm{m}$ in water $(7 \mu \mathrm{m}$ for a $400 \mathrm{keV}$ deuteron), forward directed deuterons are stopped within the water stream ${ }^{32}$. These deuterons cause D-D fusion reactions and produce neutrons within the water stream. This is therefore effectively a point source of neutrons. By contrast, backward directed deuterons move away from the main stream and interact with the gas/plasma with rapidly decreasing density, which transitions to the background density (corresponding to a pressure of approximately 20 Torr) where they have a much longer stopping distance. We estimated that at a background density in the chamber of $6.4 \times 10^{17} \mathrm{~cm}^{-3}$, a $400 \mathrm{keV}$ deuteron has a stopping distance of $30 \mathrm{~cm}^{32}$, similar to the chamber dimensions. Backward-moving deuterons therefore contribute to neutron production as a volumetric source. The importance of these neutrons is illustrated in Fig.4, which displays the neutron flux produced by forward and backward moving deuterons as well as the sum of the two. The experimental data are also plotted for comparison. Table I provides more detail such as the number of deuterons per shot, the neutron yield per deuteron and neutron yield per shot for forward and backward moving deuterons. The dominant neutron source is fusion from backward moving deuterons and consequently the larger fraction of neutrons have a volumetric origin. It has to be kept in mind, however, that we assumed complete stopping of the backward-directed deuterons. In reality, some of them would not be stopped by the low-density background gas. Thus, their contribution 
TABLE I. Simulation result table

\begin{tabular}{|c|c|c|c|c|c|c|}
\hline Intensity $\left(\mathrm{W} / \mathrm{cm}^{2}\right)$ & Energy $(\mathrm{mJ})$ & \# Deuterons $>10 \mathrm{keV}$ & $\mathrm{T}(\mathrm{keV})$ & $\begin{array}{c}\text { Neutron yield } \\
(\text { neutrons/ions) }\end{array}$ & $\begin{array}{c}\text { Neutron yield } \\
\text { (neutrons/shot) }\end{array}$ & \\
\hline \multirow{2}{*}{$1 \times 10^{19}$} & \multirow{2}{*}{3.7} & $1.4 \times 10^{9}$ & 18 & $4.0 \times 10^{-9}$ & 6 & Forward \\
& & $3.3 \times 10^{9}$ & 45 & $18 \times 10^{-9}$ & 59 & Total \\
\hline \multirow{2}{*}{$2 \times 10^{19}$} & \multirow{2}{*}{7.3} & $2.6 \times 10^{9}$ & 30 & $9.5 \times 10^{-9}$ & 25 & Forward \\
& & $5.0 \times 10^{9}$ & 55 & $26 \times 10^{-9}$ & 130 & Total \\
\hline \multirow{2}{*}{$3 \times 10^{19}$} & \multirow{2}{*}{11} & $3.7 \times 10^{9}$ & 35 & $12 \times 10^{-9}$ & 44 & Forward \\
& & $6.7 \times 10^{9}$ & 65 & $138 \times 10^{-9}$ & 255 & Total \\
\hline
\end{tabular}

is likely overestimated. The volumetric nature of the neutrons was confirmed by the neutron time-of-flight measurements, which showed a delay and increased width of the signal, which can only be interpreted as neutrons coming from different locations.

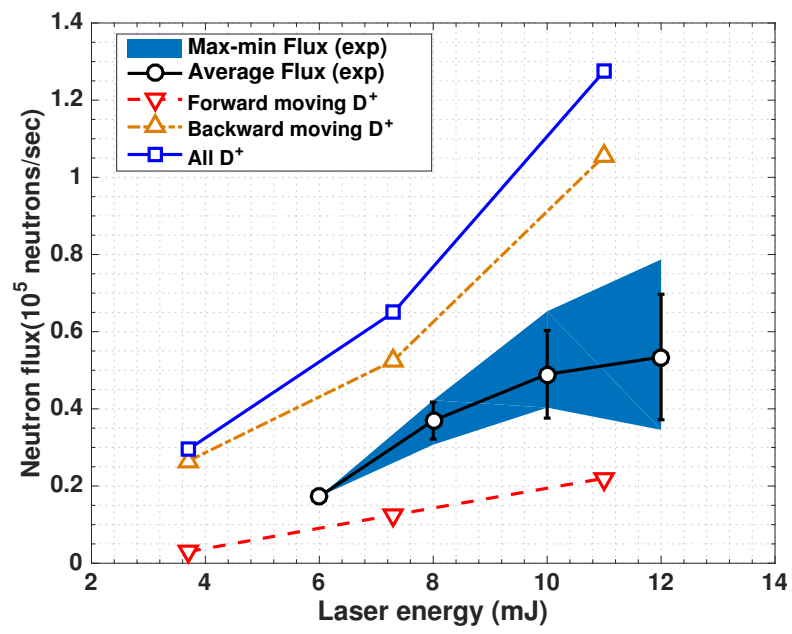

FIG. 4. Neutron flux as a function of energy from the experiments and simulations.

In conclusion, the production of D-D fusion neutrons has been demonstrated with a millijoule level femtosecond laser system. Notably, the system operates at $1 / 2 \mathrm{kHz}$ repetition rate and requires neither the replacement of deuterated targets nor a catcher alignment. These features enable the generation of a neutron flux $\left(\sim 10^{5}\right.$ neutrons/sec) that is comparable to other table-top laser based neutron sources, but for continuous all-day operation. Although the $\lambda^{3}$ system operates at $1 / 2 \mathrm{kHz}$, present technology may enable operation at $10 \mathrm{kHz}$ or higher, suggesting that such laser driven sources should be able to generate $10^{6}$ to $10^{7}$ neutrons/sec.

\section{ACKNOWLEDGMENTS}

The authors would also like to thank Shaun Clarke and the University of Michigan Neutron Science Laboratory for use of the D-D generator. This material is based upon work supported by the Air Force Office of Scientific Research under award numbers FA9550-12-1-0310 (Young Investigator Program) and FA9550-14-1-0282.

${ }^{1}$ V. Kononov et al., Nucl. Instr. Meth. Phys. Res. A 564, 525 (2006).

${ }^{2}$ M. Roth et al., Phys. Rev. Lett. 110, 044802 (2013).

${ }^{3}$ L. Perkins et al., Nucl. Fusion 40, 1 (2000).

${ }^{4}$ F. Floux et al., Phys. Rev. A 1, 821 (1970).

${ }^{5}$ T. Ditmire et al., Nature 386, 54 (1997).

${ }^{6}$ G. Pretzler et al., Phys. Rev. E 58, 1165 (1998).

${ }^{7}$ P. A. Norreys et al., Plasma Phys. Control. Fusion 40, 175 (1998).

${ }^{8}$ T. Ditmire et al., Nature 398, 489 (1999).

${ }^{9}$ L. Disdier, J. Garconnet, G. Malka, and J. Miquel, Phys. Rev. Lett. 82, 1454 (1999).

${ }^{10}$ J. Zweiback et al., Phys. Rev. Lett. 84, 2634 (2000).

${ }^{11}$ S. Karsch et al., Phys. Rev. Lett. 91, 015001 (2003).

12 J. M. Yang et al., J. Appl. Phys. 96, 6912 (2004).

${ }^{13}$ T. Žagar, J. Galy, J. Magill, and M. Kellett, New J. Phys. 7, 253 (2005).

${ }^{14}$ L. Willingale et al., Phys. Plasmas 18, 083106 (2011).

${ }^{15}$ B. Hou et al., Phys. Plasmas 18 (2011).

${ }^{16}$ C. Zulick et al., Appl. Phys. Lett. 102 (2013).

${ }^{17}$ S. Fritzler et al., Phys. Rev. Lett. 89, 165004 (2002).

${ }^{18}$ T. Nayuki et al., Rev. Sci. Instrum. 74, 3293 (2003).

${ }^{19}$ S. Ter-Avetisyan et al., Phys. Plasmas 12, 012702 (2005).

${ }^{20}$ G. Grillon et al., Phys. Rev. Lett. 89, 065005 (2002).

${ }^{21}$ K. W. Madison et al., Phys. Plasmas 11, 270 (2004).

${ }^{22}$ M. Gherendi et al., J. Optoelectron. Adv. Mater. 10, 2092 (2008).

${ }^{23}$ H. Ing, Radiat. Meas. 33, 275 (2001).

${ }^{24}$ S. Bastiani et al., Phys. Rev. E 56, 7179 (1997).

${ }^{25}$ S. Lee et al., J. Korean Phys. Soc. 51, 1695 (2007).

${ }^{26}$ P. McKenna et al., Laser Part. Beams 26, 591 (2008).

${ }^{27}$ T. Z. Esirkepov et al., Nucl. Instr. Meth. Phys. Res. A 745, 150 (2014).

${ }^{28}$ J. T. Morrison et al., Phys. Plasmas 22 (2015).

${ }^{29}$ G. M. Petrov and J. Davis, Phys. Plasmas 18 (2011).

${ }^{30}$ G. M. Petrov and J. Davis, Commun. Comput. Phys. 16, 599 (2014).

${ }^{31}$ C. Orban et al., Phys. Plasmas 22, 023110 (2015).

32 J. F. Ziegler, M. Ziegler, and J. Biersack, Nucl. Instr. Meth. Phys. Res. B 268, 1818 (2010). 


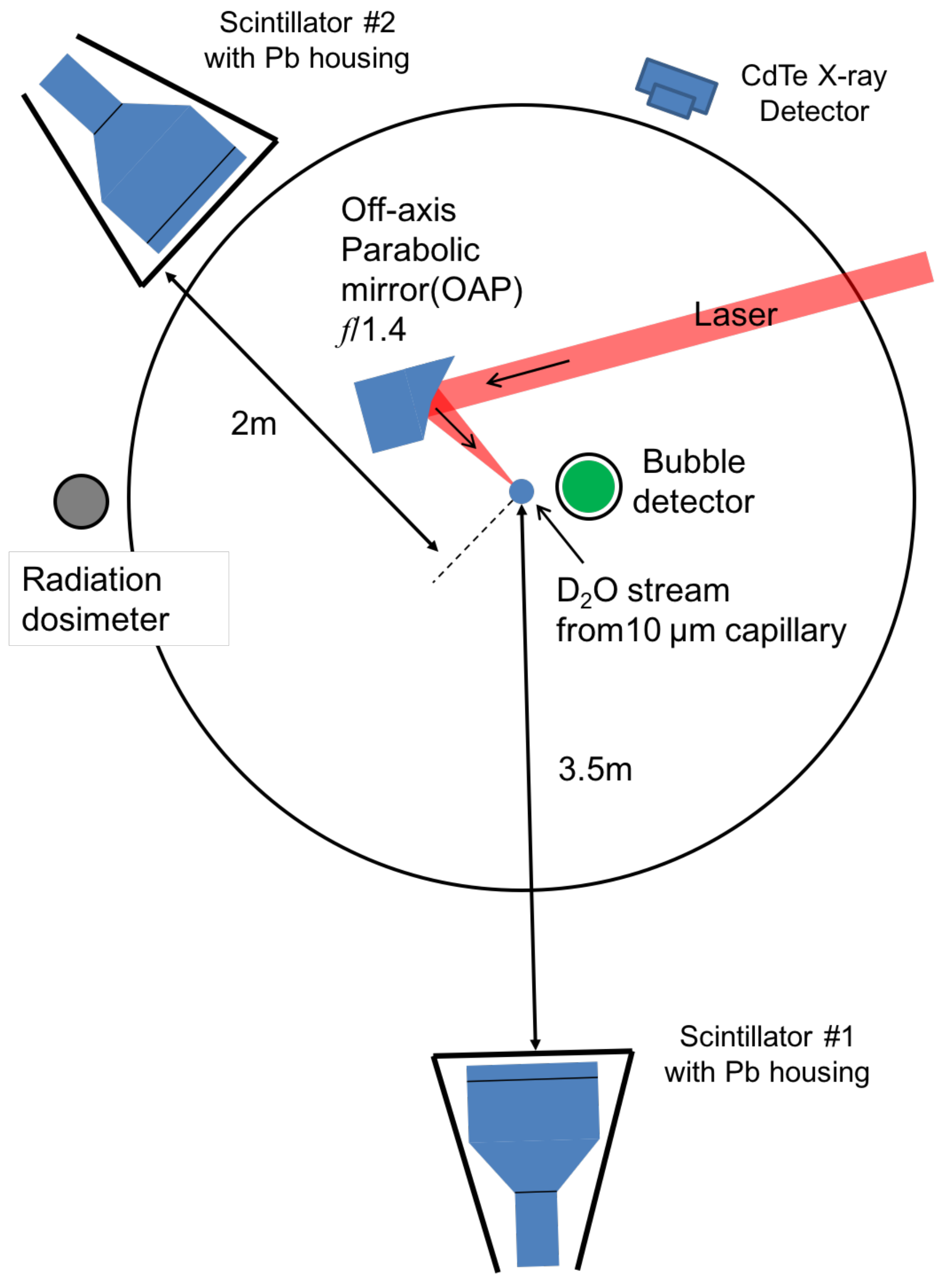




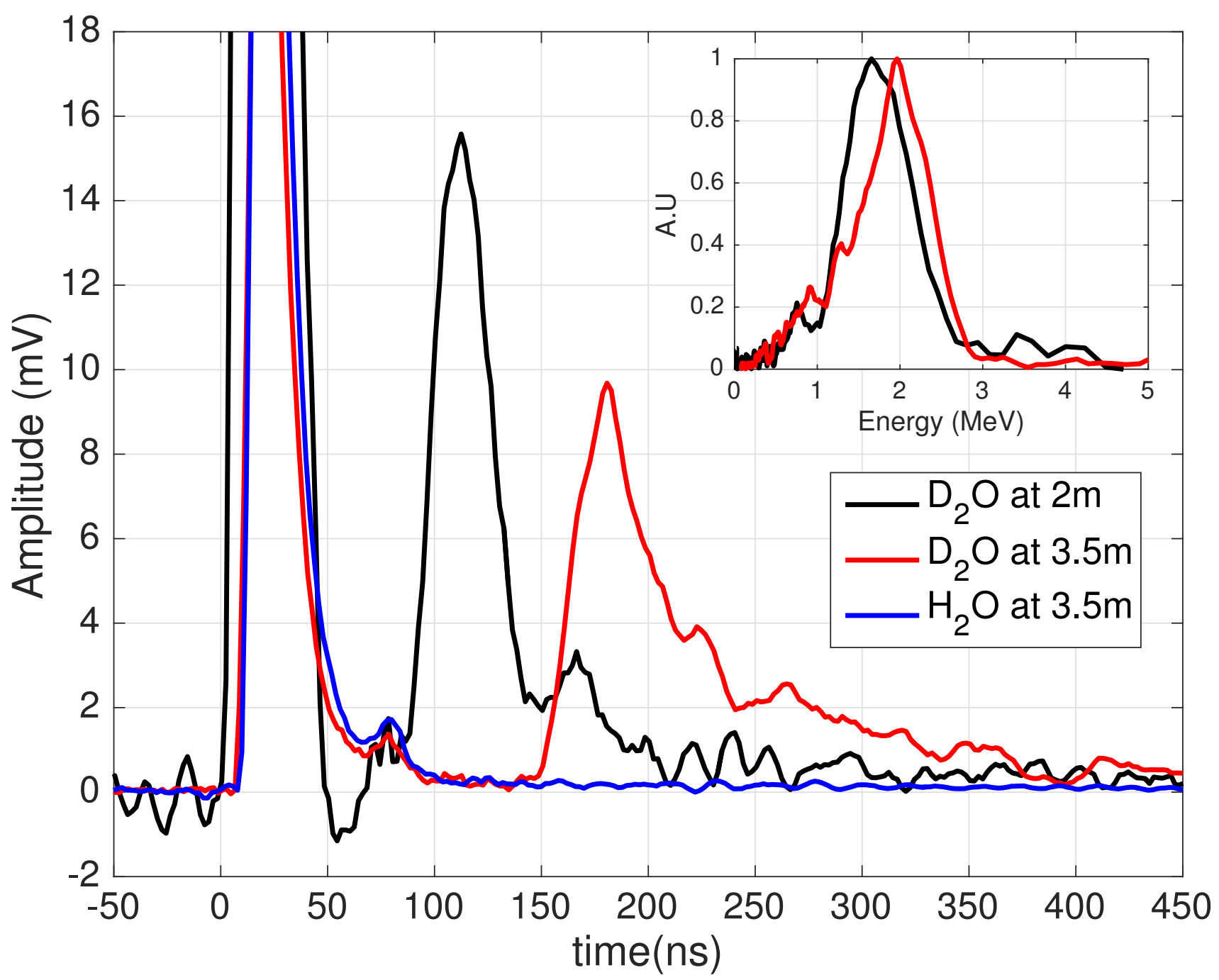




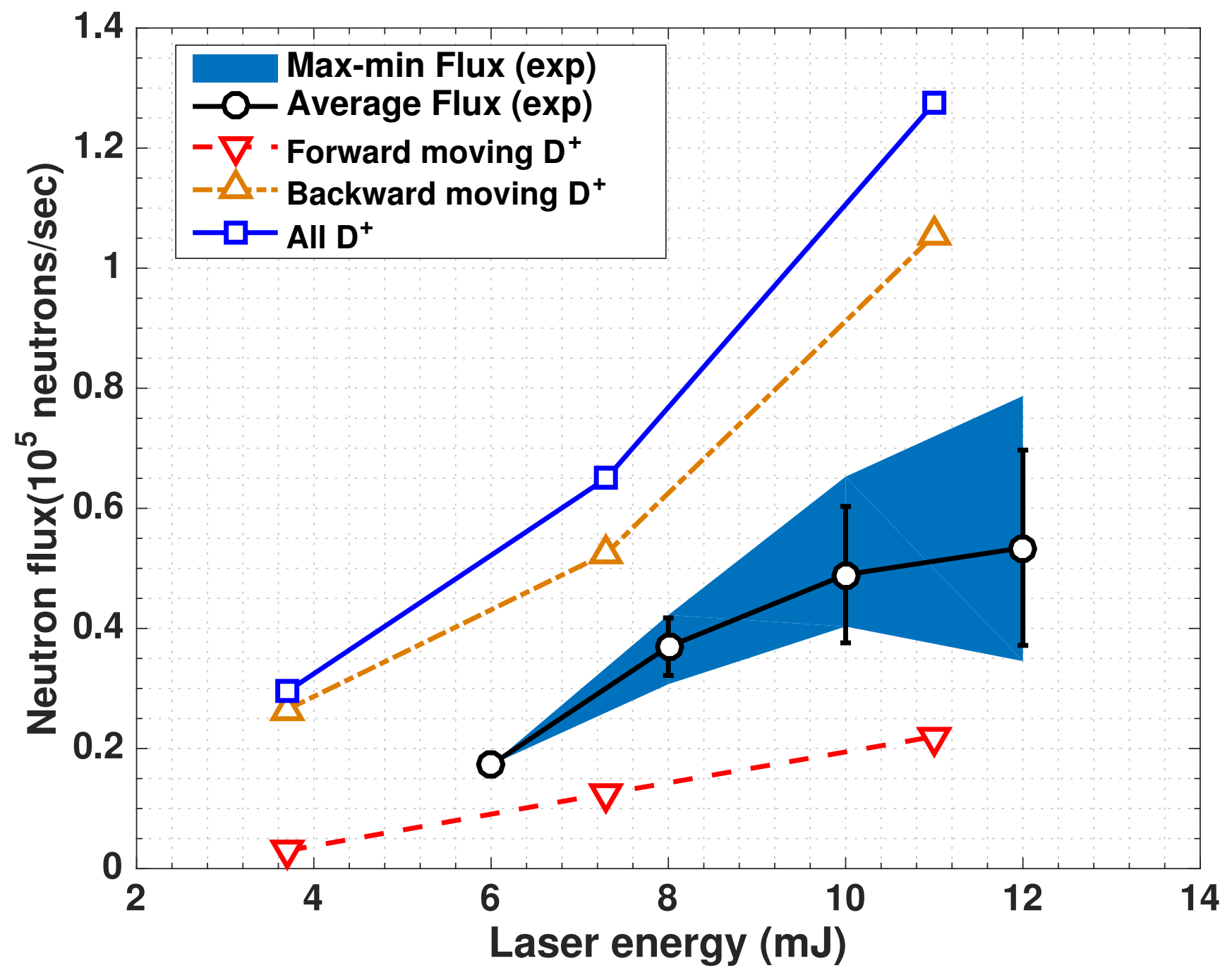

